
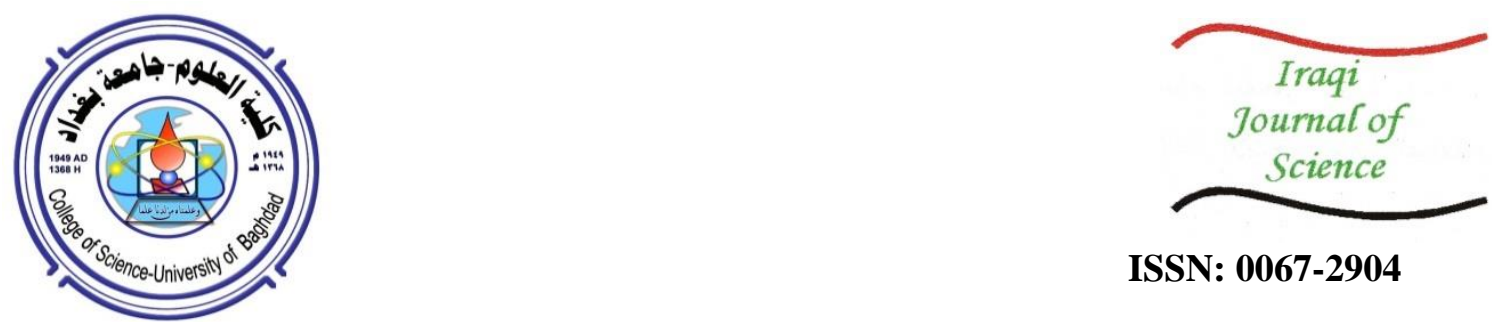

ISSN: 0067-2904

\title{
Evaluation of PARP-1 by immunohistochemistry in a sample of Iraqi patients with gastric cancer
}

\author{
Raghad Khalid Mwafaq ${ }^{1 *}$, Amal Khudair Abbas ${ }^{2}$, Leith Abdul Hussein Abdullah ${ }^{3}$ \\ ${ }^{1,2}$ Department of Biology, College of Science, University of Baghdad, Iraq \\ ${ }^{3}$ Consultant Gastroenterologist and Heptologist, Baghdad Hospital, Medical City, Baghdad, Iraq
}

Received: $25 / 4 / 2021$

Accepted: $12 / 7 / 2021$

\begin{abstract}
PARP-1 is a protein enzyme with a major role in DNA repair that is overexpressed in many malignancies. It is correlated with susceptibility and metastasis to lymph nodes in gastric cancer (GC). The objective of the present investigation is to estimate PARP1 expression in patients with gastric cancer and detected if it could be used as a predictive marker. Furthermore, we aimed to find the correlation between PARP1 expression and clinicopathological parameters, such as gender, age, invasion depth, histopathological type, involvement of lymph nodes, grade, and stages of GC. This is a retrospective study from the period 2018-2020. Fifty randomly selected subjects (10 normal and $40 \mathrm{GC}$ ) were examined for formalin-fixed, paraffin-embedded blocks (FFPE) of stomach tissue . The diagnosis reports were collected from the Pathology Department of the Gastroenterology and Hepatology Teaching Hospital and some private hospitals in Baghdad, Iraq. Hematoxylin and eosin (H\&E) and immunohistochemical (IHC) staining of PARP1 were applied for the histological sections. Statistical analysis was accomplished by SPSS system at $\mathrm{P}<0.05$. There were significant differences between the patients and control groups in the expression level of PARP1. There were also significant correlations between PARP1 expression and each of the histopathological subtype, grade, invasion depth, involvement of lymph node, and stages in patients. However, non- significant associations were found between the expression and the age and gender of patients. These results indicate that PARP1 could be employed as a good prospective marker for gastric cancer.
\end{abstract}

Keywords: PARP1, Immunohistochemistry, gastric cancer, clinicopathological parameters.

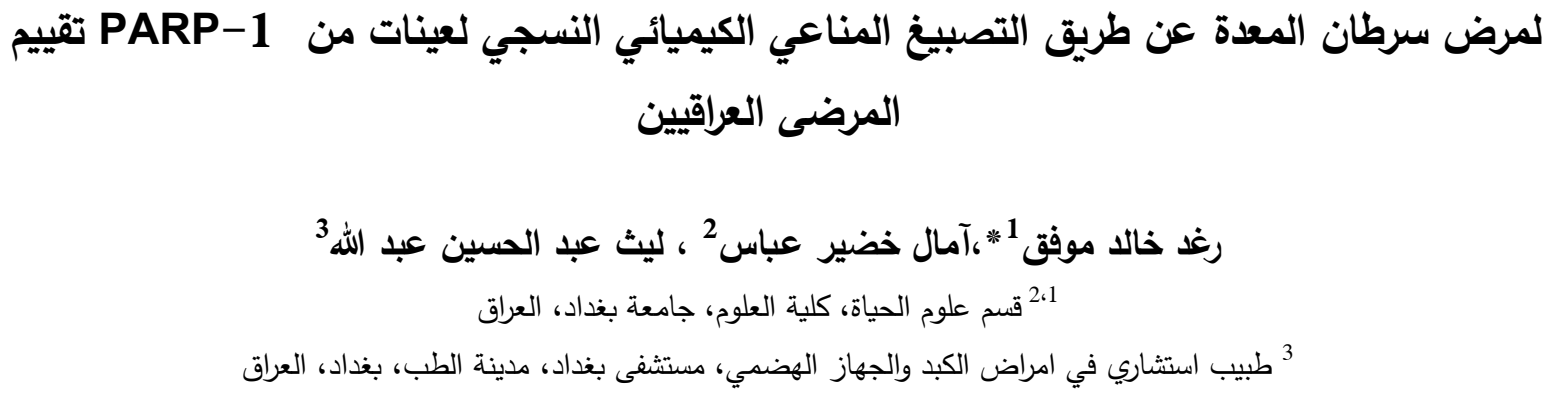

*Email: raghad_ahm77@yahoo.com 


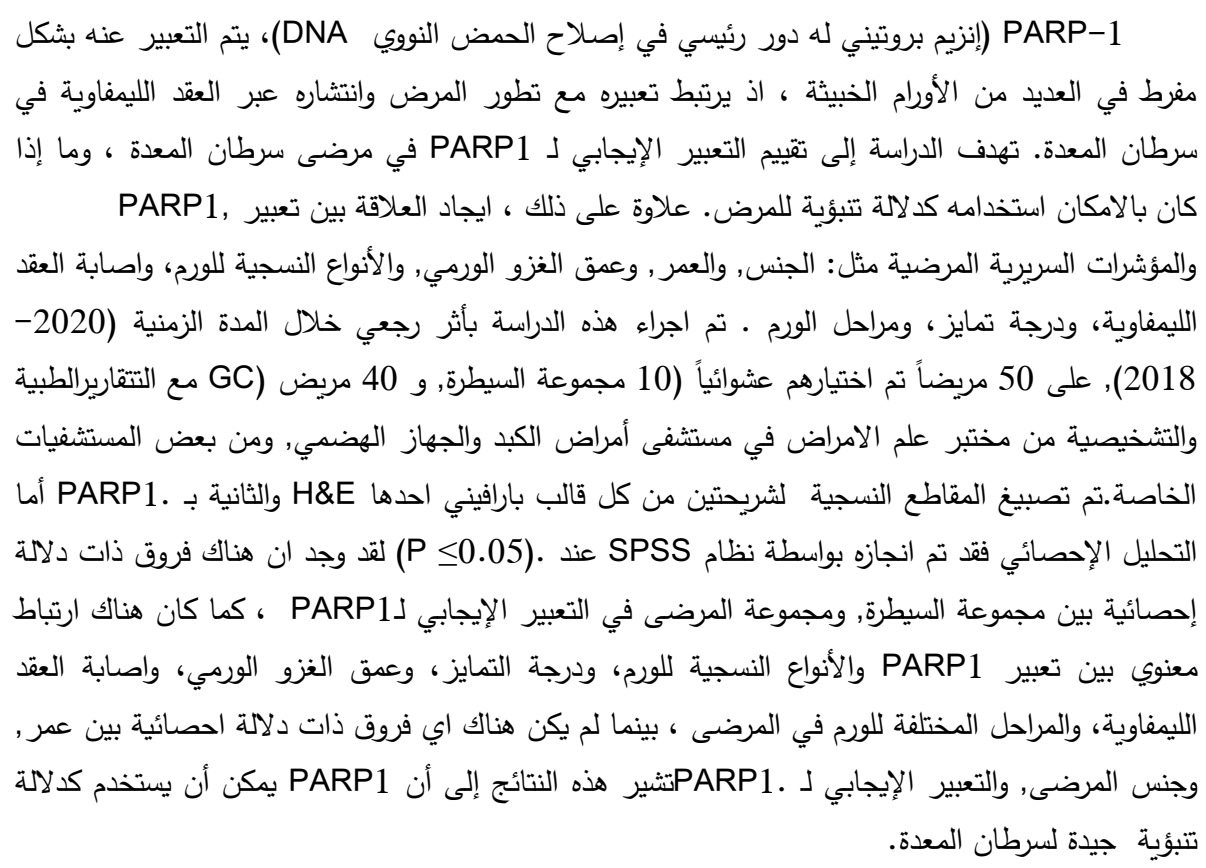

\section{Introduction}

Gastric carcinoma (GC) comprises a universal health issue. It is a malignant disease with high aggressiveness and heterogeneous nature. It is one of the most prevalent reasons for cancer- related mortality and takes advantage of an important encumbrance on international health sponsors [1]. In Iraq malignant neoplasms represent the second leading cause of death. Gastric cancer trend demonstrated an instant rise after 2007; it is ranked as the fifth of eighteen cancer types in Iraq [2]. Gastric cancer is the third main reason of cancer-related mortality in the world, leading to about 783,000 deaths in 2018 and over 1,000,000 new cases per year [3]. The Lauren classification is the most commonly utilized classification of GC. It includes three main subtypes: intestinal, diffuse and, mixed [4]. These subtypes differ in a number of properties, such as clinical characteristics, genetics, morphology, epidemiology, and development [5]. Poly (adenosine diphosphate-ribose) polymerase (PARP1) is an enzyme [6] that has many important functions in DNA damage, repair, and many cellular processes [7]. It binds to single or double stranded DNA forms; its effectiveness is mostly demanded to maintain the integrity of the genome [8]. PARP-1 is overexpressed in many types of tumors, comprising malignant melanomas, colorectal cancer, breast cancer, testicular tumors, and lymphangioleiomyomatosis. Also, it is correlated with invasiveness and poor clinical prognosis [9]. Subsequently, PARP-1 may be a possible anti-cancer target [10]. The inhibitors of PARP are also actually used in mixture with chemotherapeutic agents to raise the responses of tumor [11]. It is correlated with susceptibility and lymph node metastasis in gastric cancer [12]. Le et al. [13] stated that PARP-1 inhibitors induce the cisplatin cytotoxicity in human gastric cancer cells. Liu et al. [14] previously declared that Cochinchina momordica seed extraction significantly decreases the survival rate of human gastric malignant cells, by downregulating PARP expression. However, it remains to be determined whether the PARP-1 expression levels are associated with the tumorigenesis and progression of gastric cancer. Hence, other researchers [15] stated that PARP-1 protein expression patterns need additional study in patients with gastric cancer. The objective of the present study is to estimate PARP1 expression in Iraqi patients with gastric cancer and detect if it could be used as a predictive marker. Furthermore, we aimed to find the correlation between PARP1expression and clinicopathological parameters, such as gender, age, invasion depth, histopathological type, involvement of lymph nodes, grade, and stages of GC. 


\section{Materials and methods \\ The collection of the samples}

This study is a retrospective investigation of data recorded in the period from January 2018 to December 2020. Fifty randomly-selected subjects (10 normal and 40 GC) were examined for formalin-fixed, paraffin-embedded blocks (FFPE) of stomach tissue. The diagnosis reports were collected for surgically resected specimens in the Pathology Department, Gastroenterology and Hepatology Teaching Hospital, Medical City/ Baghdad/Iraq after obtaining the official approval from the Iraq Ministry of Health, Department of Medical City/ Baghdad/ Iraq. No chemotherapy was received by these patients. Each report contained clinical-pathological parameters, i.e. age, gender, histopathological type, grade, depth of invasion, lymph nodes involvement, and tumor, node, and metastasis (TNM), which were diagnosis by pathological doctors of hospital. The group of forty patients included 24 males and 16 females, while the control group involved 2 males and 8 females. These ten samples of normal stomach tissues were selected randomly from patients undergoing sleeve gastrectomy in private hospitals. These cases were classified depending on Lauren classification [4].

\section{Staining}

Each FFPE was cut in $5 \mu \mathrm{m}$ thickness before staining, mounted on positively charged slides for IHC and normal slides for H\&E. Some of the sections for the control and patient groups were stained by routine H\&E staining based on Suvarna et al. [16]. PARP1-antibody (mouse monoclonal antibody; catalog \#MAB 8095; dilution 1/100; R\&D, USA) was used in this study to evaluate the immunohistochemistry expression by using R\&D protocol. For H\&E staining, the sections put in xylene for 2 times, then in descending serial solutions of ethyl alcohol. The slides were washed by tap water and then stained by Harris's hematoxylin and eosin to stain the nucleus and cytoplasm, respectively. The slides were then cleared by xylene, mounted by dibutylphthalate polystyrene xylene (DPX), covered by cover slides, and then dried by electric oven at $45 \mathrm{C}^{\circ}$. For IHC, the sections were incubated in serum-blocking solution, incubated with (PARP1- antibody), and then incubated with biotinylated link secondary antibody (Abcam, USA). The slides were incubated with streptavidin-enzyme conjugate (Thermo Fisher, USA) and then incubated with substrate-chromogen 3,3'Diaminobenzidine (DAB), which is often used in immunohistochemical staining as a chromogen mixture (Abcam, USA). Finally, the slides were stained with hematoxylin and mounted with an aqueous mounting solution. After each of these steps, the slides were washed with PBS buffer to remove excess material.

\section{Negative and positive control}

The positive control of this antibody was breast cancer tissue, as in R\&D protocol, in which the nucleus of the cell gives a brown color when the histological section is treated by this primary antibody (Figure 1), whereas the section from breast cancer tissue without adding primary antibody was used as negative control, in which the nucleus is not stained with brown color (Figure 2).

\section{Scoring system}

Colored cancer cells were calculated by selecting four areas. The total selected cancer cells were not less than 100 cells, which were examined at magnification powers of $10 \mathrm{X}$ and 40X. Colored cancer cells were calculated in hall slide. Cells with brown color in nucleus were considered to be positive, whereas uncolored cells were considered as negative. Depending on a previously described method [17], the scoring system was accomplished and has the following scores: score 0: $0 \%$, score $1:<25 \%$, score $2: 25-50 \%$, score $3:>75 \%$.

\section{Statistical analysis}

Data analysis was accomplished by using the statistical package for social science/SPSS version - 24 software/ IBM. Chi - square (X2) was used to estimate the correlation between 
clinical-pathological properties and the expression of the marker and compered with control group. Differences with P-values at $\leq 0.05$ were considered as significant.

\section{Results}

From 50 patients included in this study, the results indicated that males total number in the control group was 2, while that of females was 8 . In the gastric cancer group, the total number of males was 24 , while that of females numbers was 16 . The age of the subjects in the control group ranged 19-50 years, comprising one age group of equal or less than 50 years, with a mean age (of $33.9 \pm 10.027$ years. The age of patients with gastric cancer ranged 19-83 years with a mean value of $55.325 \pm 15.423$ years. The patients group was divided into two age groups, one equal to or less than 50 years and the other is more than 50 years. The number of patients who were equal or less than 50 years old was $16(40 \%)$ with a mean age of 39.875 \pm 9.493 years. The numbers of patients who were more than 50 years old was $24(60 \%)$ with a mean age of $65.625 \pm 8.234$ years. The histopathological subtyping showed that the patients were distributed to $23(57.5 \%)$ cases of the intestinal type, $15(37.5 \%)$ cases of the diffuse type, and $2(5 \%)$ cases of the mixed type. Based on the clinicopathological parameters, the patients were divided into $25(62.5 \%)$ moderately differentiated cases and $15(37.5 \%)$ poorly differentiated cases of the total number of patients. The current study showed that gastric cancer invasion in the subserosa was observed in $15(37.5 \%)$ cases; invasion in the serosa was in $18(45 \%)$ cases, while muscularis propria invasion was in $7(17.5 \%)$ cases of the total patients. The total number of patients diagnosed with lymph node involvement was about 32 (80\%), whereas patients without lymph node involvement had a total number of $8(20 \%)$. The invasive depth and the TNM staging system were used to distribute patients with gastric cancer into advanced stages (III \& IV) and early stage (II). Advanced stages total numbers of patients was $27(67.5 \%)$ cases, divided into $12(30 \%)$ cases in the III stage and $15(37.5 \%)$ cases in the IV stage. The total number of patients in the early stage was $13(32.5 \%)$ (Table $1)$.

Table 1- Study groups distribution depending on clinicopathological parameters

\begin{tabular}{|c|c|c|c|}
\hline Clinicopathological Parameters & & ings & Frequency (\%) \\
\hline \multirow{4}{*}{ Gender } & \multirow{2}{*}{ Control } & Male & $2(20 \%)$ \\
\hline & & Female & $8(80 \%)$ \\
\hline & \multirow{2}{*}{ Patients } & Male & $24(60 \%)$ \\
\hline & & Female & $16(40 \%)$ \\
\hline \multirow{4}{*}{ Age } & \multirow{2}{*}{$\leq 50$} & Control & $10(100 \%)$ with mean $(33.9 \pm 10.027)$ \\
\hline & & Patients & $16(40 \%)$ with mean $(39.875 \pm 9.493)$ \\
\hline & \multirow{2}{*}{$>50$} & Control & - \\
\hline & & Patients & $24(60 \%)$ with mean $(65.625 \pm 8.234)$ \\
\hline \multirow{3}{*}{ Histopathological types } & \multirow{3}{*}{ Patients } & Intestinal & $23(57.5 \%)$ \\
\hline & & Diffuse & $15(37.5 \%)$ \\
\hline & & Mix & $2(5 \%)$ \\
\hline \multirow{2}{*}{ Grade of tumor } & \multirow{2}{*}{ Patients } & Moderately & $25(62.5 \%)$ \\
\hline & & Poorly & $15(37.5 \%)$ \\
\hline \multirow{2}{*}{ Invasion depth of tumor } & \multirow{2}{*}{ Patients } & pT2 & $7(17.5 \%)$ \\
\hline & & pT3\& pT4 & $33(82.5 \%)$ \\
\hline \multirow{2}{*}{ Involvement of lymph node } & \multirow{2}{*}{ Patients } & N0 & $8(20 \%)$ \\
\hline & & $\mathrm{N} 1,2 \& 3$ & $32(80 \%)$ \\
\hline \multirow{2}{*}{ Stage of GC (TNM) } & \multirow{2}{*}{ Patients } & II & $13(32.5 \%)$ \\
\hline & & III\&IV & $27(67.5 \%)$ \\
\hline
\end{tabular}




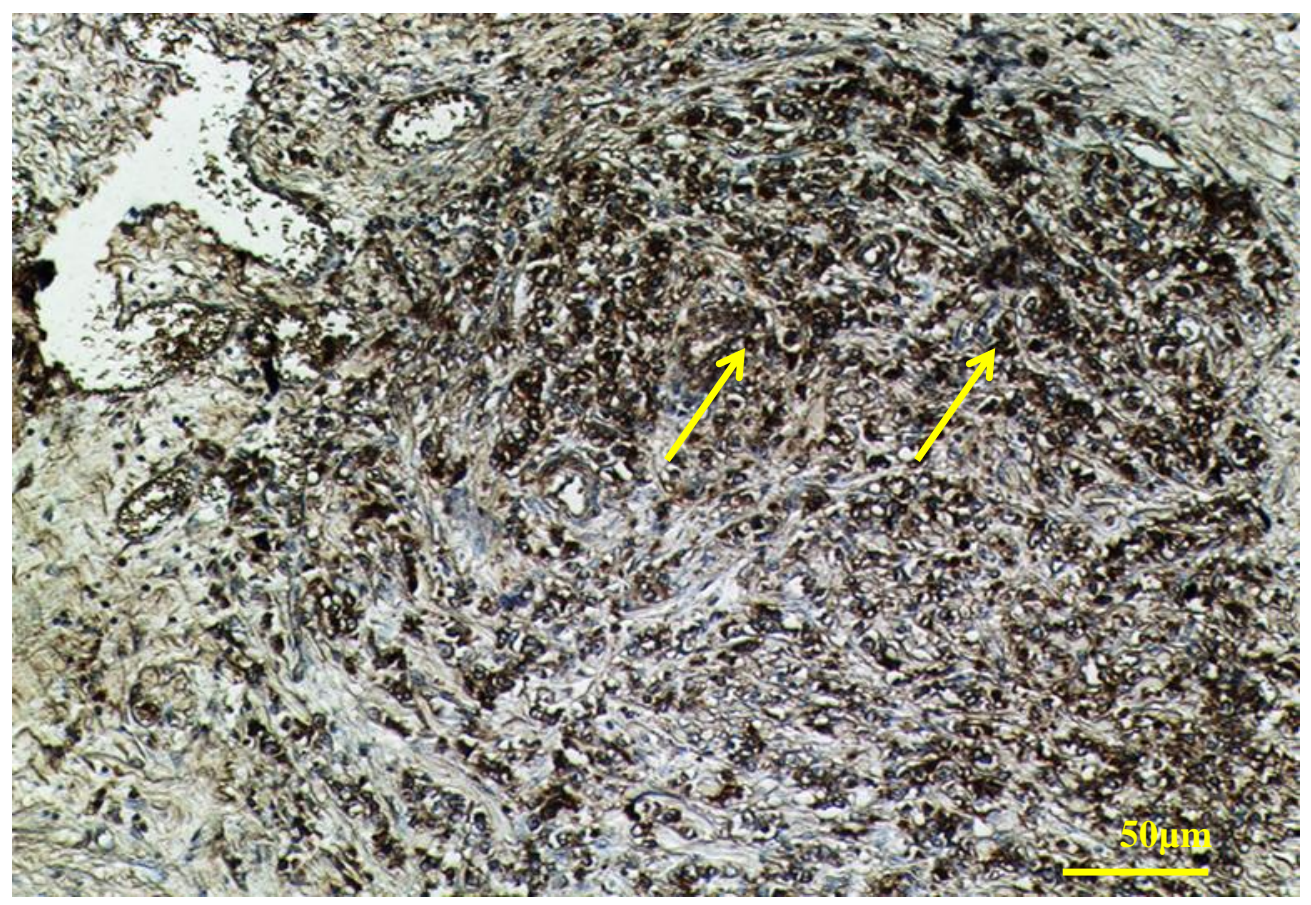

Figure 1- Cross section of IHC stained breast cancer tissue in the positive control group; nuclei are stained in brown color (yellow arrow); scale bar $50 \mu \mathrm{m}, 40 \mathrm{X}$.

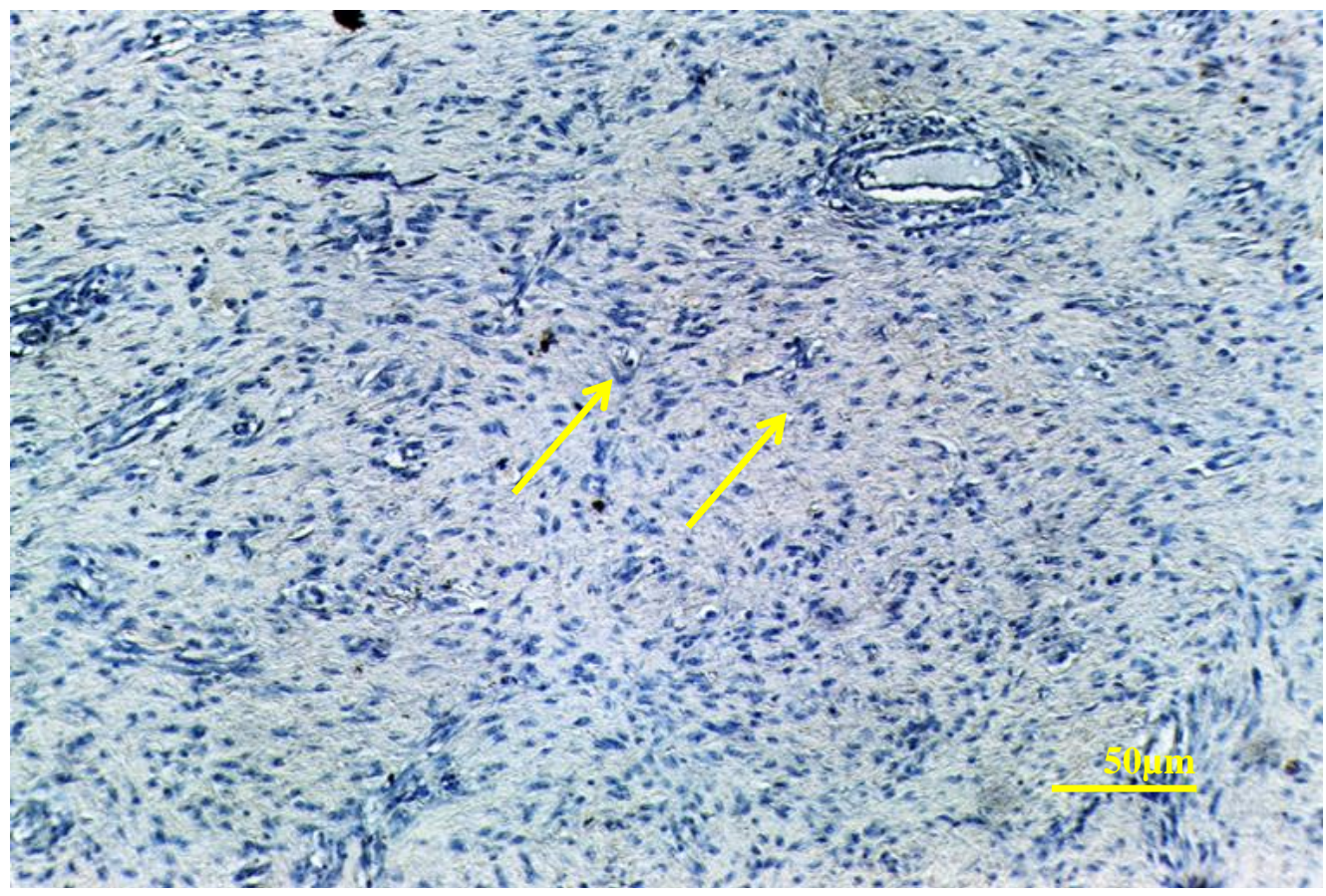

Figure 2-Cross section in IHC-stained breast cancer tissue of the negative control group; nuclei are not stained in brown color (yellow arrow); scale bar $50 \mu \mathrm{m}, 40 \mathrm{X}$.

\section{The IHC expression of PARP1}

The current study indicated that PARP1 is expressed in high levels in patients with gastric cancer only, reaching a number of 40 (100\%), whereas the control group showed no expression in any case. In patients with gastric cancer, score 0 was recorded in $2(5 \%)$ cases, while the number of patients with score +1 was $8(20 \%)$. Also, $8(20 \%)$ cases had score +2 and $22(55 \%)$ cases had score +3 . This differences in the expression levels between two 
groups have led to statistically significant associations at $\mathrm{P} \leq 0.05$ and $\mathrm{P}=0.0001$, as shown in Table 2 and Figures 3-8.

Table 2-The expression of PARP1 in patients with gastric cancer as compared to the control group

\begin{tabular}{|c|c|c|c|c|}
\hline \multicolumn{2}{|c|}{ Expression of PARP1 } & $\begin{array}{c}\text { Patients with gastric } \\
\text { cancer } \\
\text { number (ratio) }\end{array}$ & $\begin{array}{c}\text { Control } \\
\text { number (ratio) }\end{array}$ & \multirow{2}{*}{ P- value } \\
\hline \multirow{2}{*}{ Negative } & Score 0 & $2(5 \%)$ & $10(100 \%)$ & \\
\cline { 2 - 4 } & Score +1 & $8(20 \%)$ & $0(0 \%)$ & \multirow{2}{*}{ P $=0.0002^{\mathrm{s}}$} \\
\cline { 2 - 4 } Positive & Score +2 & $8(20 \%)$ & $0(0 \%)$ & \multirow{2}{*}{0} \\
\cline { 2 - 4 } & Score +3 & $22(55 \%)$ & $0(0 \%)$ & \\
\hline \multicolumn{2}{|c|}{ Total number/ number (ratio) } & $40(100 \%)$ & $10(100 \%)$ & \\
\hline
\end{tabular}

S: Chi- square; Differences are significant at $\mathbf{P} \leq \mathbf{0 . 0 5}$.

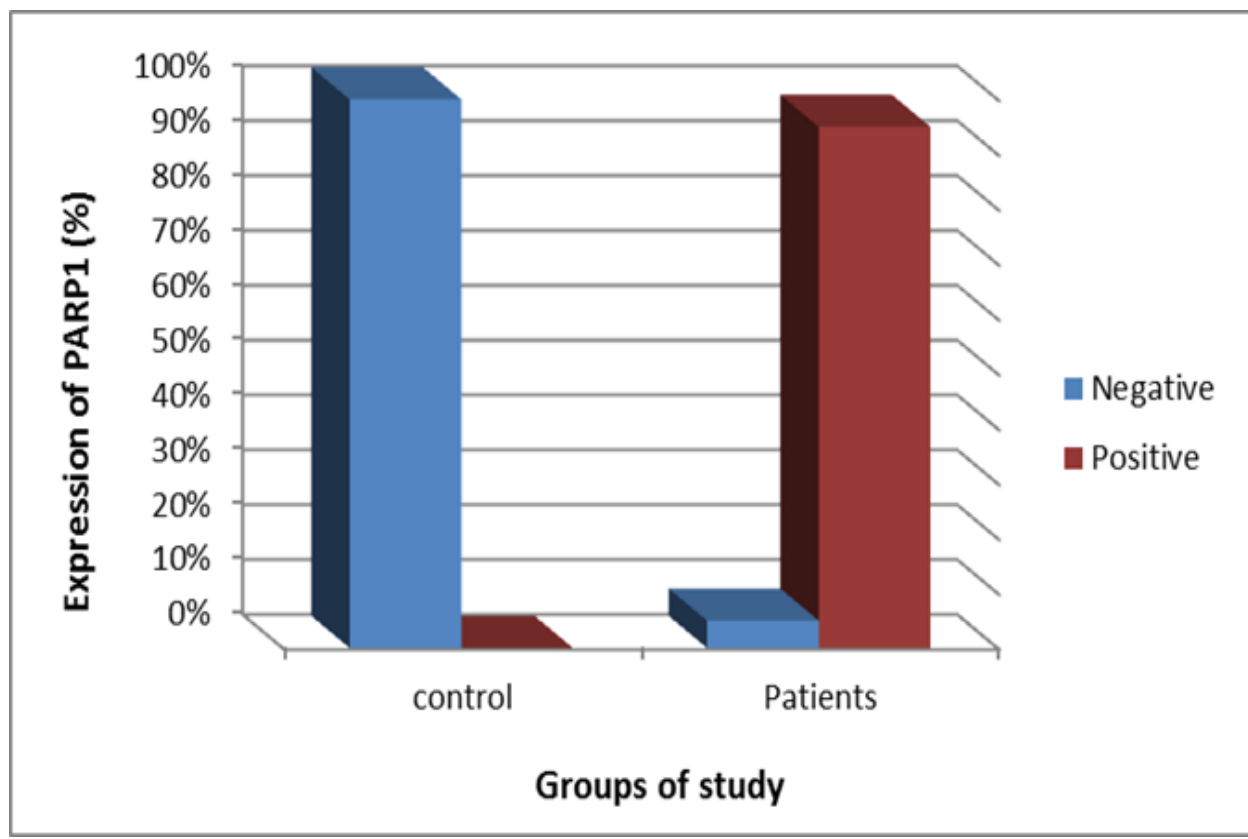

Figure 3-Graph illustrates the expression of PARP1 in patients with gastric cancer as compared to the control. 


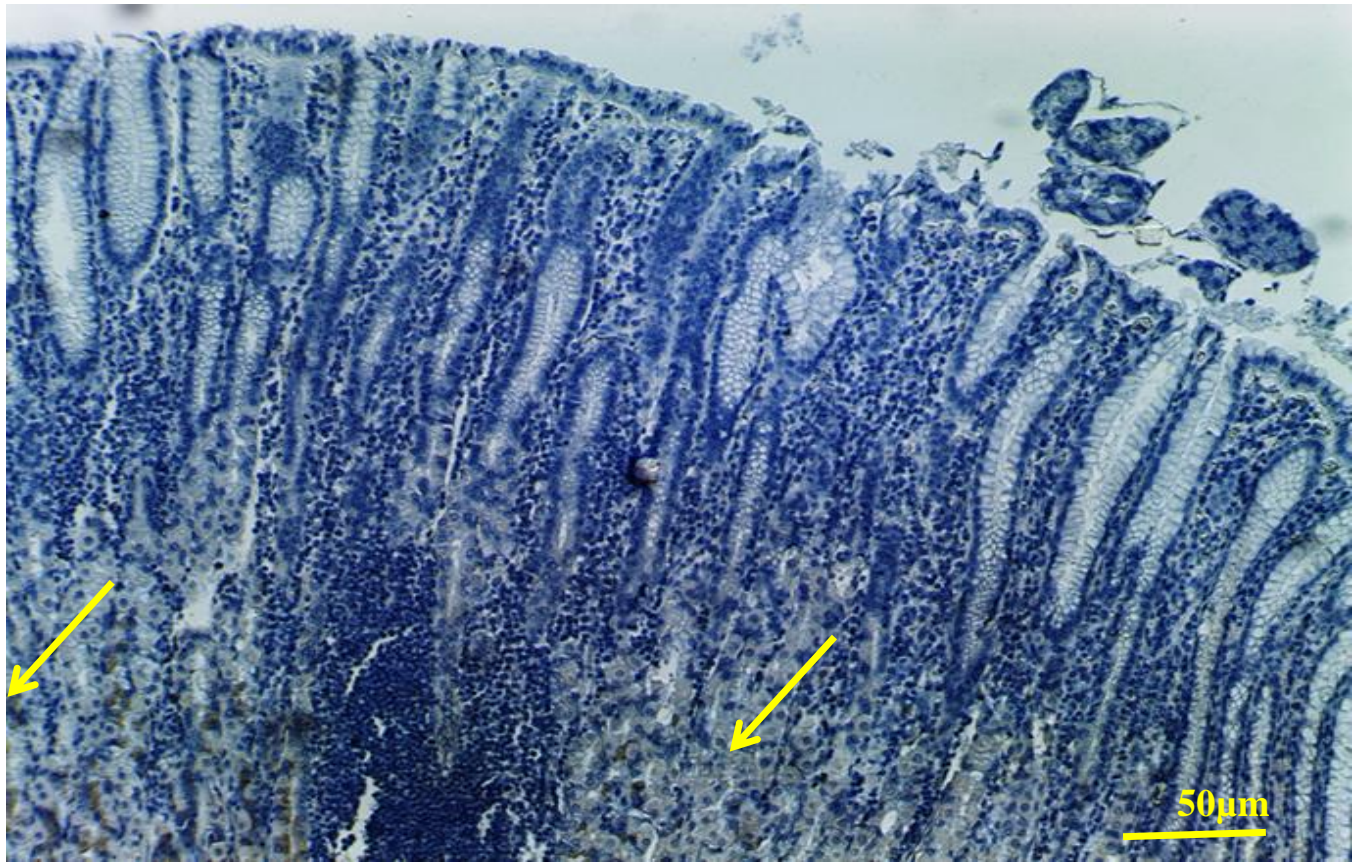

Figure 4-Control IHC-stained stomach tissue illustrating the negative expression of PARP1 in gastric cancer patients with score 0; no nuclei are stained in brown color (arrow) (scale bar $50 \mu \mathrm{m}, 40 \mathrm{X})$.

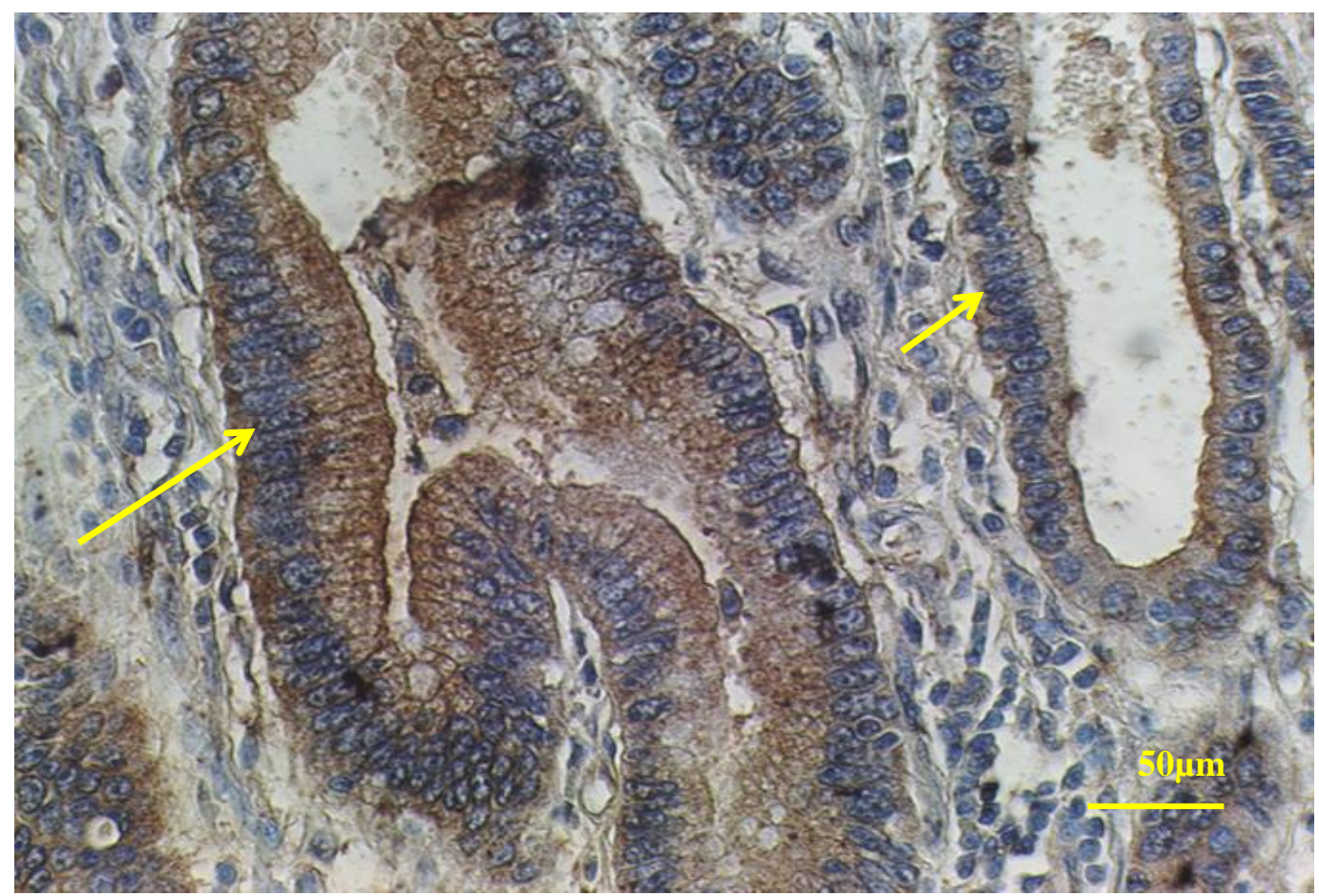

Figure 5-IHC-stained gastric cancer tissue illustrating the negative expression of PARP1 in gastric cancer patients with score 0 ; no nuclei are stained in brown color (arrow) (scale bar 50 $\mu \mathrm{m}, 40 \mathrm{X})$. 


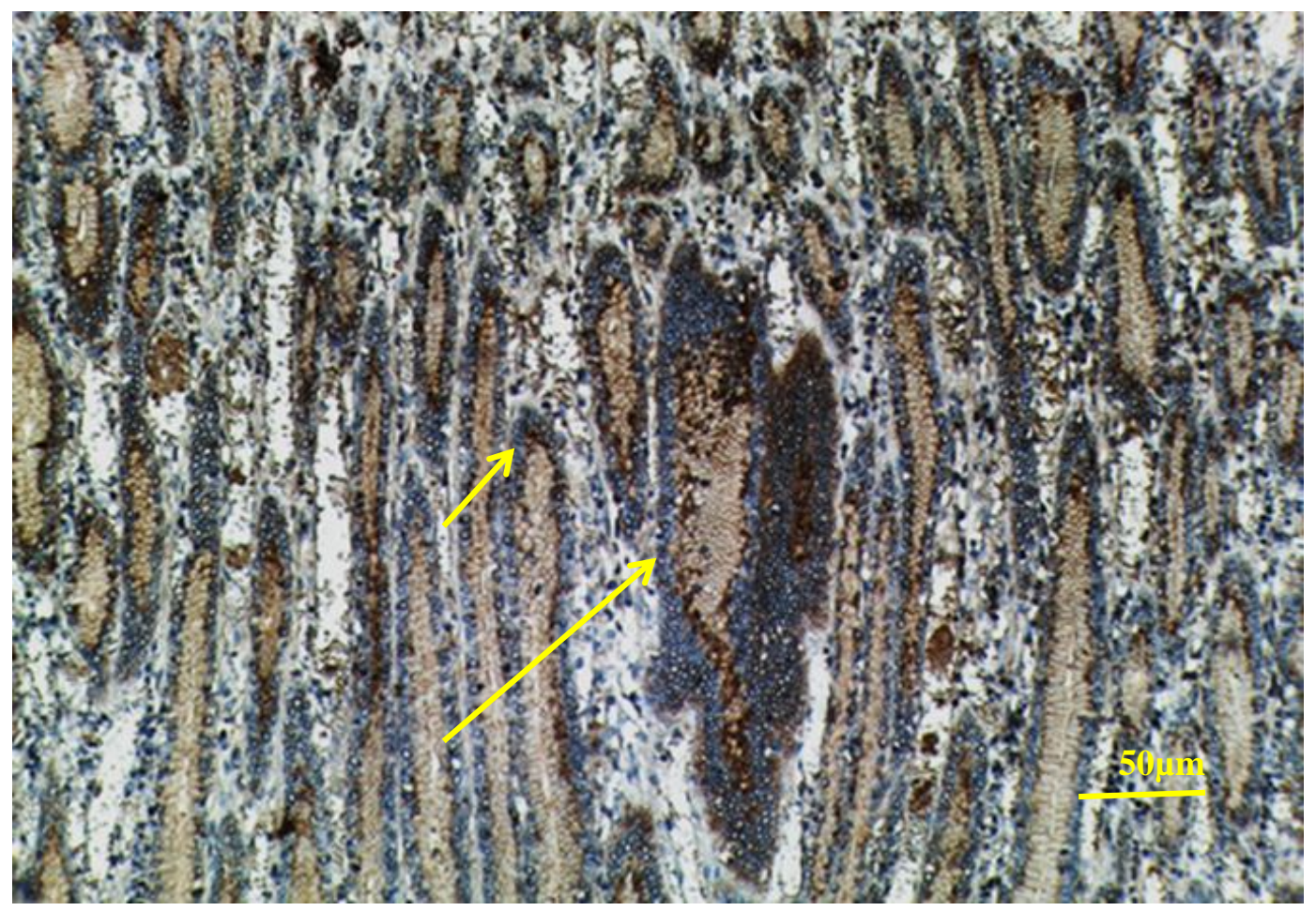

Figure 6-Cross section in IHC-stained gastric cancer stomach wall (moderately differentiated intestinal subtype) illustrating positive expression of PARP1 (arrow) in Score +1 patients; < $25 \%$ of nuclei are weakly stained in brown color. Scale bar $50 \mu \mathrm{m}, 40 \mathrm{X}$.

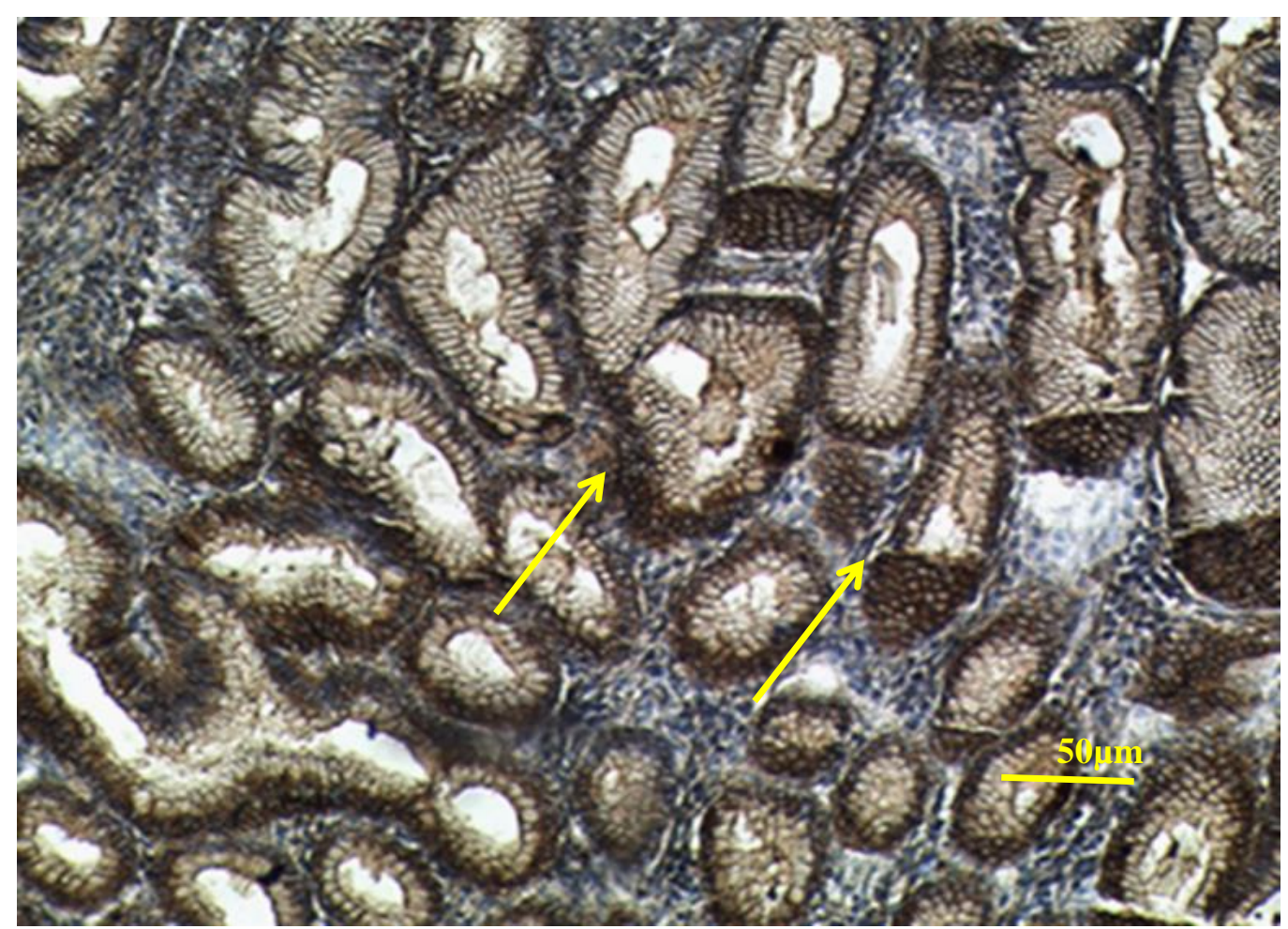

Figure 7-Cross section in IHC-stained gastric cancer stomach wall (moderately differentiated intestinal subtype) illustrating positive expression of PARP1 (arrow) Score +2 patients; 25$50 \%$ of nuclei are moderately stained in brown color. Scale bar $50 \mu \mathrm{m}, 40 \mathrm{X}$. 


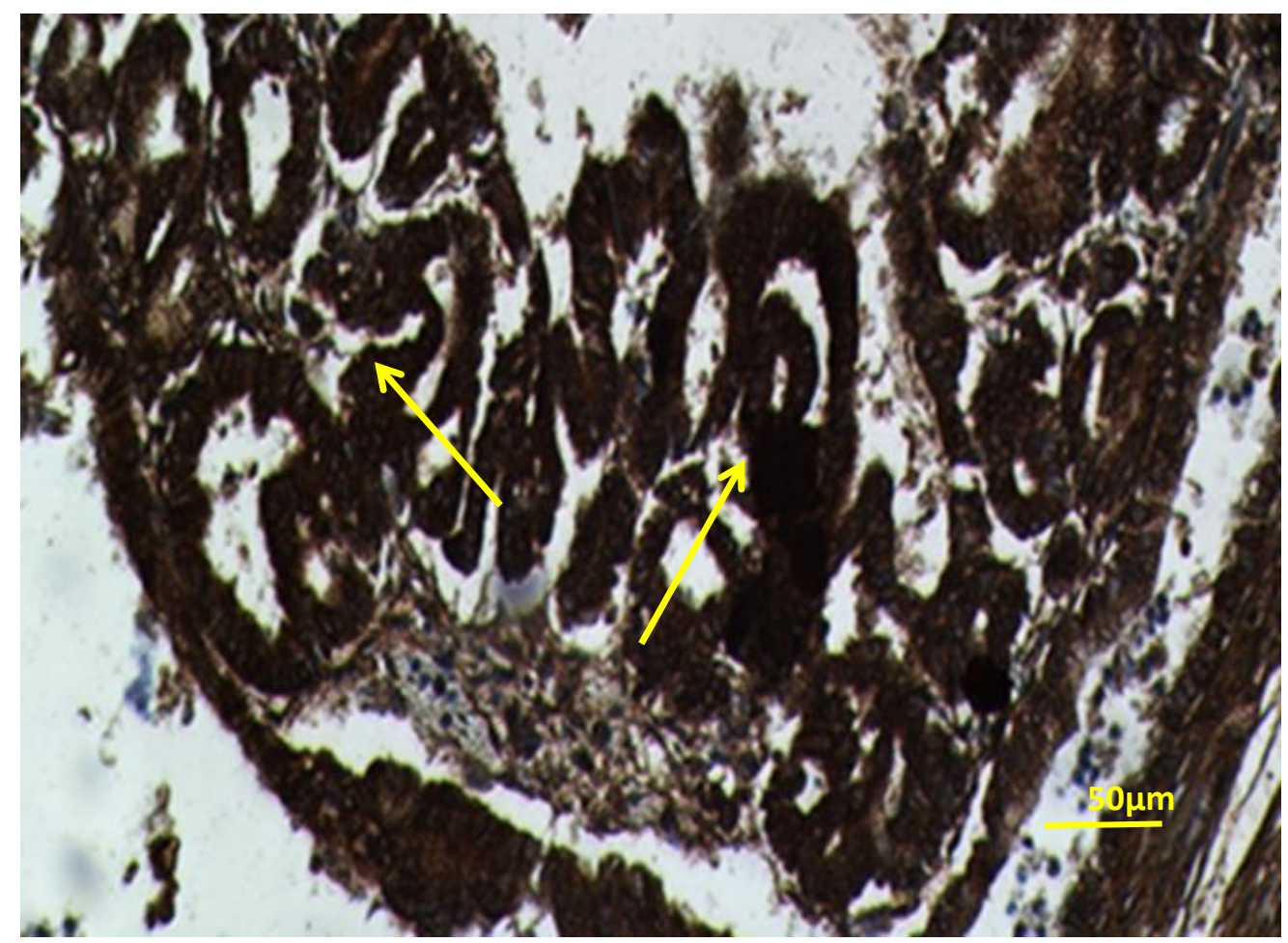

Figure 8-Cross section in IHC-stained gastric cancer human stomach wall (intestinal subtype, moderately differentiated) illustrating strongly positive expression of PARP1 (arrow) in score +3 patients; $>75 \%$ of nuclei are stained in brown color. Scale bar $50 \mu \mathrm{m}, 40 \mathrm{X}$.

Table 3 shows the expression of PARP1 in correlation with clinicopathological parameters. This study indicates that $55 \%$ of the total number of patients with gastric cancer had positive expressed. Positive expression was recorded in 22 cases of males and 16 cases of females. , with no significant association according to gender. The age of the entire group ranged 19-83 years. The expression of PARP1was recorded in $27.5 \%$ of the patients who are less or equal to 50 years old and $57.5 \%$ of the patients who are more than 50 years old. The P-value of 0.608 indicated no significant association between age and the expression of this marker. The current study showed that the percentage of patients with the intestinal type who had positive expression was $62.5 \%$, with the diffuse was $37.5 \%$, and with the mixed type was $5 \%$, with significant associations at $\mathrm{P} \leq 0.05$, as shown in Table 3 . The expression of PARP1 was found in $57.5 \%$ of the moderately differentiated grade patients and $37.5 \%$ of those with poorly differentiated grade. The positive expression of PARP1in patients according to invasion depth was recorded in $17.5 \%$ in pT2 and (82.5\%) in pT3 and 4, with a significant association at $\mathrm{P} \leq 0.05$. Also, the current study indicated that PARP1 positive expression was found in $80 \%$ of patients with lymph nodes involvement (N1, N2, and N3), whereas the value in patients without involvement of lymph nodes (N0) was 15\%. Statistically, these differences showed a significant association between the involvement of lymph nodes and the expression of PARP1. The highest records of PARP1 positive expression were found in $67.5 \%$ of patients with the advanced stages of disease (III and IV, while it was 27.5\%) in the early stage. Statistically, these differences showed a significant association between the stage of gastric cancer and the expression of PARP1 at $\mathrm{p} \leq 0.05$. 
Table 3-The expression of PARP1 associated with clinicopathological parameters

\begin{tabular}{|c|c|c|c|c|}
\hline \multirow{2}{*}{\multicolumn{2}{|c|}{ Clinicopathological parameters }} & \multicolumn{2}{|c|}{ Expression of PARP1 } & \multirow[b]{2}{*}{ P-value } \\
\hline & & $\begin{array}{c}\text { Negative } \\
\text { Number (ratio) }\end{array}$ & $\begin{array}{c}\text { Positive } \\
\text { Number (ratio) }\end{array}$ & \\
\hline \multirow{2}{*}{ Gender } & Male & $2(5 \%)$ & $22(55 \%)$ & \multirow{2}{*}{$\mathrm{P}=0.496^{\mathrm{NS}}$} \\
\hline & Female & $0(0 \%)$ & $16(40 \%)$ & \\
\hline \multirow{2}{*}{ Age } & $\leq 50$ & $1(2.5 \%)$ & $15(27.5 \%)$ & \multirow{2}{*}{$\mathrm{P}=0.608^{\mathrm{NS}}$} \\
\hline & $>50$ & $1(2.5 \%)$ & $23(57.5 \%)$ & \\
\hline \multirow{3}{*}{$\begin{array}{l}\text { Histopathological } \\
\text { subtype }\end{array}$} & Intestinal & $2(5 \%)$ & $21(62.5 \%)$ & \multirow{3}{*}{$\mathrm{P}=0.023^{\mathrm{S}}$} \\
\hline & Diffuse & $0(0 \%)$ & $15(37.5 \%)$ & \\
\hline & Mix & $0(0 \%)$ & $2(5 \%)$ & \\
\hline \multirow{2}{*}{ Grade of tumor } & Moderately & $2(5 \%)$ & $23(57.5 \%)$ & \multirow{2}{*}{$\mathrm{P}=0.034^{\mathrm{S}}$} \\
\hline & Poorly & $0(0 \%)$ & $15(37.5 \%)$ & \\
\hline \multirow{2}{*}{$\begin{array}{c}\text { Invasion depth of } \\
\text { GC }\end{array}$} & pT 2 & $2(5 \%)$ & $7(17.5 \%)$ & \multirow[b]{2}{*}{$\mathrm{P}=0.00004^{\mathrm{S}}$} \\
\hline & pT3 \& pT4 & $0(0 \%)$ & $33(82.5 \%)$ & \\
\hline \multirow{2}{*}{$\begin{array}{l}\text { Involvement of } \\
\text { lymph node }\end{array}$} & No & $2(5 \%)$ & $6(15 \%)$ & \multirow{2}{*}{$\mathrm{P}=0.00051^{\mathrm{S}}$} \\
\hline & $\mathrm{N} 1,2, \& 3$ & $0(0 \%)$ & $32(80 \%)$ & \\
\hline \multirow{2}{*}{$\begin{array}{l}\text { Stage of GC } \\
\text { (TNM) }\end{array}$} & II & $2(5 \%)$ & $11(27.5 \%)$ & \multirow{2}{*}{$\mathrm{P}=0.00001^{\mathrm{S}}$} \\
\hline & III\&IV & $0(0 \%)$ & $27(67.5 \%)$ & \\
\hline
\end{tabular}

Chi- square; is the differences are significant at $\mathbf{P} \leq \mathbf{0 . 0 5}$.

\section{Discussion}

In Iraq, malignant neoplasms represent the second leading cause of death. Gastric cancer trend demonstrated an instant rise after 2007, being ranked as the fifth among eighteen cancer types in Iraq [2]. GC comprises a universal health issue. It is a malignancy disease with a highly aggressive nature. It is one of the most prevalent reasons for cancer- related death and takes advantage of an important encumbrance on international health sponsors [1]. Options of gastric cancer treatment are restricted because of its heterogeneity and genetic complication [18]. Therefore, finding special biomarkers is important for managing the development of gastric cancer and identifying effective treatments for patients [19]. This study indicated that the number of male was higher than that of female patients. Also, the number of older patients (more than 50 years) was higher than that of younger ones (equal or less than 50 years). Similarly, the study of Lou et al. [20] showed that men are at a higher incidence than women. Furthermore, the maximal sex difference was recorded in the older age. Moreover, the GLOBOCAN 2012 report demonstrated that the standardized rates of the age of gastric cancer patients were twice higher in men [21]. The study of Radkiewicz et al. [22] demonstrated that the rise in the risk of gastric cancer is associated with the male sex. The portion of cancer explicated by factors correlated to male sex is huge. Males also suffer poorer survival from cancer in most of the studied locations. A probable exposition is either the preventative impact of estrogen in women or other effects as diversity in diet and occupational exposure, $[23,24]$. Many studies stated that the intestinal type of the disease is the most commonly found type, with a rising risk of adenocarcinoma, in the age range of 55-80 years, being more commonly appearing in males than female (in the ratio of 2:1) [25, 26]. Furthermore, the current study found that about $57.5 \%$ of the cases were in the age of more than 50 years. These results correspond to the study of Sattar and Al Mallah [27], which indicated that about $67.1 \%$ of cases were in the age range of 40 to 69 years. Also, in another study [28], the author reported that $54 \%$ of cases were of the intestinal subtype, i.e. located in the distal stomach (non- cardia), associated with infection by H. pylori. All these results have consistency with the present study, in which the intestinal type had the highest ratio compared with the diffuse 
and mix types. This study also recorded that the moderately differentiated subtype was found in a higher ratio than the poorly differentiated subtype, which has similarity to the results of another study [29]. These results also correspond to those of Ashour et al., [30], who stated that the moderately differentiated subtype was the most common among all cases (about 63.3\%). In contrast, the study of Zhang et al. [31] found that the poorly differentiated subtype was dominant. Advanced stage (III and IV), based on TNM staging analysis, showed a higher ratio than the early stage (II), which result agrees with Chen et al. [32, 33]. The IHC analysis of the correlation between the expression of PARP1 and the clinicopathological parameters shows no significant association with age and gender, whereas there was a significant correlation with lymph node involvement. The results of TNM staging and invasion depth of GC , but not those of the grade of differentiation, corresponded with those of Abd- Allah et al.[34] . Moreover, these results agree with those of Park et al. and Liu et al [35, 36]. There was also a significant association in the current study between the histopathological type and the expression of this marker, which agrees with the study of Park et al. [37]. Wang et al. [38] found that the activity of PARP1 in the tumor cells of a patient with gastric cancer causes cisplatin resistance. If PARP1 is inhibited, this can induce cisplatin, which stimulates the damage of DNA and apoptosis. These results reflect the possibile clinical importance of PARP1 activity in GC. Furthermore, the function of PARP1 in cancer development and metastasis is demonstrated in the repair of DNA and regulation of transcription in several oncogenes, such as hypoxia-inducible factor $1 \alpha$ and 2A genes and Vascular Endothelial Growth Factor Receptor 1 (VEGFR1) gene [39]. Also, it regulates gene expression by various mechanisms, such as remodeling of chromatin, methylation of DNA, and RNA polymerase II [40]. The stimulation of G2/M cell cycle arrest, but not apoptosis, was caused by PARP1 inhibition, which implies that the expression of PARP1 has an important role in GC metastasis [37]. In conclusion, these results indicated that PARP1 could be a good prospective marker for gastric cancer.

\section{Acknowledgements}

All thanks and appreciation to all members of the Gastroenterology and Hepatology Teaching Hospital and some private hospitals in Baghdad for all help to accomplish this study.

\section{References}

[1] J.P. Gao, W. Xu, W.T. Liu, M. Yan and Z.G. Zhu, "Tumor heterogeneity of gastric cancer: From the perspective of tumor-initiating cell", World J. Gastroenterol, vol.24, no. 24, pp. 2567-2581, 2018.

[2] A.AM. Hussain and R.K. Lafta, "Cancer Trends in Iraq 2000-2016", Oman Med J. vol.36, no.1, pp. e219, 2021.

[3] A.P. Thrift and H.B. El-Serag, "Burden of gastric cancer", Clin Gastroenterol.Hepatol, vol.18, no. 3, pp. 534-542, 2020.

[4] P. Lauren, "The two histological main types of gastric carcinoma: diffuse and so-called intestinaltype carcinoma: an attempt at a histoclinical classification", Acta Pathol Microbiol Scand., vol.64, pp. 31-49,1965.

[5] J. Machlowska, J. Baj, M. Sitarz, R. Maciejewski and R. Sitarz, "Gastric Cancer: Epidemiology, Risk Factors, Classification, Genomic Characteristics and Treatment Strategies", Int J Mol Sci., vol.21, no. 11, pp. 4012, 2020.

[6] A. Ray Chaudhuri and A. Nussenzweig, "Review: The multifaceted roles of PARP1 in DNA repair and chromatin remodeling", Nat Rev Mol Cell Biol.vol.18, no.10, pp. 610-621, 2017.

[7] E.E. Alemasova and O.I. Lavrik, "Review: Poly (ADP-ribosyl) ation by PARP1: reaction mechanism and regulatory proteins", Nucleic Acids Res., vol. 47, no.8, pp.3811-3827, 2019.

[8] M.G. Bacalini, D. Di Lonardo, A. Catizone, F. Ciccarone, T. Bruno, M. Zampieri, T. Guastafierro, R. Calabrese, M. Fanciulli, C. Passananti, P. Caiafa and A. Reale, "Poly (ADP- 
ribosyl) ation affects stabilization of Che-1 protein in response to DNA damage", DNA Repair (Amst)., vol.10, no. 4, pp $380-9,2011$.

[9] Y. Sun, D. Gallacchi, E.Y. Zhang, S.B. Reynolds, L. Robinson, I.A. Malinowska, T.T. Chiou, A.M. Pereira, C. Li, D.J. Kwiatkowski, P.S. Lee, J.J. Yu, "Rapamycin-resistant poly (ADPribose) polymerase-1 overexpression is a potential therapeutic target in lymphangioleiomyomatosis", Am J Respir Cell Mol Biol., vol. 51, no. 6, pp738-49, 2014.

[10] A.N. Weaver and E.S. Yang, "Beyond DNA Repair: Additional Functions of PARP-1 in Cancer", Front Oncol., vol.3, pp290, 2013.

[11] S. Benafif and M. Hall, "An update on PARP inhibitors for the treatment of cancer", Onco Targets Ther., vol.8, pp 519-28, 2015.

[12] W. He, T. Liu, Y. Shan, K. Zhu and Y. Li, "PARP1 polymorphisms increase the risk of gastric cancer in a Chinese population", Mol Diagn Ther.vol.16, no.1, pp.35-42, 2012.

[13] T.V. Le, J.H. Suh, N. Kim and H.J. Park. "In silico identification of poly (ADP-ribose) polymerase-1 inhibitors and their chemosensitizing effects against cisplatin-resistant human gastric cancer cells", Bioorg Med Chem Lett., vol.23, no.9, pp. 2642-2646, 2013.

[14] H.R. Liu, L.Y. Meng, Z.Y. Lin, Y. Shen, Y.Q. Yu and Y.Z. Zhu. "Cochinchina momordica seed extract induces apoptosis and cell cycle arrest in human gastric cancer cells via PARP and p53 signal pathways". Nutr Cancer, vol. 64, no.7, pp. 1070-1077, 2012.

[15] Y. Liu, Y. Zhang, Y. Zhao, D. Gao, J. Xing and H. Liu, "High PARP-1 expression is associated with tumor invasion and poor prognosis in gastric cancer", Oncol Lett., vol.12, no.5, pp. 3825$3835,2016$.

[16] S.K. Suvarna, C. Layton and J.D. Bancroft, Bancroft's theory and practice of histological techniques. Elsevier, 2019, p.169.

[17] M. Marques, M.C. Beauchamp, H. Fleury, I. Laskov, S. Qiang, M. Pelmus, D. Provencher, , A.M. Mes-Masson, W.H. Gotlieb and M. Witcher, "Chemotherapy reduces PARP1 in cancers of the ovary: implications for future clinical trials involving PARP inhibitor", BMC Medicine, no.13, pp217, 2015.

[18] F. Lordick, W. Allum, F. Carneiro, E. Mitry, J. Tabernero, P. Tan, E. Van Cutsem, C. van de Velde and A. Cervantes, "Unmet needs and challenges in gastric cancer: the way forward", Cancer Treat Rev., vol.40, no.6, pp.692-700, 2014.

[19] Y. Liu, Y. Zhang, Y. Zhao, D. Gao, J. Xing, and H. Liu, "High PARP-1 expression is associated with tumor invasion and poor prognosis in gastric cancer", Oncol Lett. Nov; vol. 12, no.5, pp. 3825-3835, 2016.

[20] L. Lou, L. Wang, Y. Zhang, G. Chen, L. Lin, X. Jin, Y. Huang and J., Chen, "Sex difference in incidence of gastric cancer: an international comparative study based on the global burden of disease study 2017", BMJ.vol.10, no.1, pp.e033323, 2020.

[21] J. Ferlay, I. Soerjomataram, R. Dikshit, S. Eser, C. Mathers, M. Rebelo, D.M. Parkin, D. Forman and F. Bray, "Cancer incidence and mortality worldwide: sources, methods and major patterns in GLOBOCAN 2012", Int J Cancer, vol.136, no.5, pp.E359-386, 2015.

[22] C. Radkiewicz, A.L.V. Johansson, P.W. Dickman, M. Lambe and G. Edgren, "Sex differences in cancer risk and survival: A Swedish cohort study", EJC., no. 84, pp. 130-140, 2017.

[23] M.C. Camargo, Y. Goto, J. Zabaleta, D.R. Morgan, P. Correa and C.S. Rabkin, "Sex hormones, hormonal interventions, and gastric cancer risk: a meta-analysis", Cancer Epidemiol Biomarkers Prev., vol.1, no.21, pp.20-38, 2012.

[24] S.H. Xie and J. Lagergren, "The Male Predominance in Esophageal Adenocarcinoma. Clinical gastroenterology and hepatology: the official clinical practice journal of the American gastroenterological association," Clinical Gastroenterology and Hepatology, vol.14, no.3, pp. 338-347, 2016.

[25] M.B. Piazuelo and P. Correa, "Gastric cancer: Overview", Colomb Med, vol.44, no.3, pp 192201, 2013.

[26] J. M. Marques-Lespier, M. Gonzalez-Pons, M. Cruz-Correa, "Current Perspectives on Gastric Cancer", Gastroenterol Clin North Am., vol. 45, no., 3, pp.413-428, 2016.

[27] S. A. Sattar and N. Al Mallah, "Study on lung infections of patients with cancer under chemotherapy", Iraqi Journal of Science, vol. 60, no.8, pp. 1682-1688, 2019. 
[28] M. Cisło, A. A. Filip, G. J. A. Offerhaus, B. Ciseł, K. Rawicz-Pruszynski, M.Skierucha and W. P. Polkowski, "Distinct molecular subtypes of gastric cancer: from Lauren to molecular pathology", Oncotarget, vol. 9, no. 27, pp. 19427-19442, 2018.

[29] Y. Zhou, J.-G. Cui, F. Huang, A. Zhang, C. Li, Z.-C. Zhao, W.-D. Li and W.-H. Fu, "Prognostic Factors For Survival In Node-Negative Gastric Cancer Patients Who Underwent Curative Resection", Scandinavian Journal of Surgery, vol. 106, no.3, pp. 235-240, 2017.

[30] H. J. Ashour, N. A. Al-Bakri and K. H. Abdul Ghafour, "The Immunohistochemical Assessment of Muc5ac in Patients with Gastric Carcinoma (Gc) in Iraq," Iraqi Journal of Science, vol. 60, no.3, pp: 460-468, 2019.

[31] W. Zhang, D. He, D. Chen, T. Li, X. Chen, K. Yang, MD, K. Liu, B. Zhang, MD, Z. Chen, Z. Zhou, and J. K. Hu, "Comparison between superficial muscularis propria and deep muscularis propria infiltration in gastric cancer patients", Medicine (Baltimore), vol.95,no.29,pp.e4165, 2016.

[32] J. Chen, R. Cai, G. Ren, J. Zhao, H. Li, C. Guo, W. He, X. Wu and W. Zhang, "Differences in clinicopathological characteristics and computed tomography findings between signet ring cell carcinoma and non-signet ring cell carcinoma in early and advanced gastric cancer", Cancer Medicine, vol.7, no. 4, pp.1160-1169, 2018.

[33] J. H. Lee, M. S. Park, K. W. Kim, J. S. Yu, M. J. Kim,S. W. Yang, "Advanced gastric carcinoma with signet ring cell carcinoma versus non-signet ring cell carcinoma: differentiation with multidetector CT"', J. Comput. Assist. Tomogr., vol.30, no.6, pp.880-884, 2006.

[34] M.S. Abd- Allah, H.M. Abd El-Kariem and, H.M. Rashad, "Evaluation of (PARP-1) Expression in Gastric Carcinoma by Immunohistochemistry and Quantitative Real-Time PCR and its Relation to HER2 Status", Med. J. Cairo Univ., vol. 88, no. 4, pp.1581-1587, 2020.

[35] S.H. Park, K.Y. Jang, and M.J. KIM, "Tumor suppressive effect of PARP1 and FOXO3A in gastric cancers and its clinical implications", Oncotarget, vol.6, no. 42, pp. 44819-44831, 2015.

[36] Y. Liu, Y. Zhang, Y. Zhao, D. Gao, J. Xing and H. Liu, "High PARP-1 expression is associated with tumor invasion and poor prognosis in gastric cancer". Oncol.Lett., vol.12, no.5, pp. 3825-35, 2016.

[37] S. Park, K.Y. Jang, M.J. Kim, S. Yoon, Y. Jo, S. M.Kwon, K.M. Kim, K.S. Kwon, C.Y. Kim and H. G.Woo., "Tumor suppressive effect of PARP1 and FOXO3A in gastric cancers and its clinical implications", Oncotarget, vol. 6, no. 42, pp. 44819-44831, 2019.

[38] Q. Wang, J. Xiong, D. Qiu, X. Zhao, D. Yan, W. Xu and J. Zhou, "Inhibition of PARP1 activity enhances chemotherapeutic efficiency in cisplatin-resistant gastric cancer cells," The International Journal of Biochemistry \& Cell Biology, no. 92, pp. 164-72, 2017.

[39] L. Wang, C. Liang, Lif., "PARP1 in Carcinomas and PARP1 Inhibitors as Antineoplastic Drugs", Int. J.Mol. Sci., vol.18, no.10, pp. 2111, 2017.

[40] Y. Fengf, J.S. De Bono, M.A. Rubin and K.E. Knudsen, "Chromatin to Clinic: The Molecular Rationale for PARP1 Inhibitor Function”, Mol. Cell, no.58, pp. 925-34, 2015. 